\title{
Las prácticas educativas parentales: análisis por género de una muestra de adolescentes Coahuilenses
}

Parental educative practices: gender analysis of an adolescent sample from Coahuila

\author{
José González Tovar \\ Alicia Hernández Montaño
}

\begin{abstract}
Resumen
El presente estudio tuvo como objetivo examinar de manera cualitativa las diferencias por género de las prácticas parentales implementadas en las familias de adolescentes. El análisis por género se realizó considerando la perspectiva de los hombres y las mujeres sobre las prácticas que realizan sus padres para educarlos. La muestra por conveniencia estuvo compuesta por 133 alumnos de nivel secundaria de la ciudad de Saltillo, Coahuila. Se aplicó un cuestionario de diez preguntas abiertas de las cuales se tomaron las dos que en particular se refieren a lo que el padre y madre hacen para educar al adolescente. El procedimiento de análisis se realizó a través del programa ATLAS.ti para datos cualitativos. En los hombres hay un elemento diferenciador en las prácticas educativas enunciadas y es el que refiere al castigo físico, el cual fue un elemento aún presente en la dinámica padre-hijo en la etapa adolescente. Las mujeres no reportaron más que la amonestación verbal como método de corrección. Los hombres reportaron tanto el castigo físico como el verbal en sus respuestas aunque no se precisó el nivel de severidad del mismo ni las características particulares de su aplicación. Se concluyó que el papel de los estereotipos en la cultura mexicana y su influencia en las prácticas de crianza justifica el trato más enérgico y poca comunicación hacia los varones, y un trato de mayor protección y cuidado, así como una comunicación más abierta que permita la expresión de las emociones en las mujeres.
\end{abstract}

\section{Abstract}

The present study aimed to examine qualitative differences by gender of parental practices implemented in families of adolescents. The analysis by gender was conducted considering the perspective of men and women about the practice that their parents made to educate them. The convenience sample consisted of 133 students of secondary school of the city of Saltillo, Coahuila. A questionnaire of ten open questions was applied from which two questions in particular were taken concerning what the father and mother do to educate adolescents. The analysis procedure was conducted through the ATLAS.ti program for qualitative data. There is a differentiating element in educational practices that refers to corporal punishment, which was an element present in the parent-child dynamic at the adolescent stage. The women reported no more than verbal reprimand as a method of punishment. It was concluded that the role of stereotypes in the Mexican culture and its influence on rearing practices justified stronger treatment and little communication towards boys, and a treatment of greater protection and care as well, a more open communication that allow the expression of the emotions in women.

Palabras Clave: prácticas parentales, género, control psicológico, control conductual.

Keywords: parental practices, gender, psychological control, behavioral control. 


\section{Introducción}

La familia constituye uno de los primeros escenarios educativos con los cuales contamos a lo largo de nuestra vida y abarca, en muchos casos, las primeras interacciones en el desarrollo psicológico de los niños; éstas últimas se extienden hasta la etapa de la adolescencia, donde el contexto familiar sigue jugando un papel crucial en el ajuste psicológico del joven (Parra \& Oliva, 2002). Es sabido que la existencia de problemas de comunicación entre padres e hijos adolescentes constituye uno de los factores familiares de riesgo más estrechamente vinculados con el desarrollo de problemas de salud mental en los hijos. También pueden influir negativamente, por ejemplo, en la presencia de síntomas depresivos, ansiedad, estrés, baja autoestima, bajo rendimiento escolar o los problemas de integración social.

En este sentido, Papalia y Wendwoks (2009) reconocen que es fundamental el papel de los padres como primera instancia para el desarrollo de una socialización adecuada y pro-social para sus hijos. Si entendemos la socialización familiar como el proceso de interacción en el contexto familiar, cuyo objetivo es inculcar en los hijos un sistema de valores, normas y creencias, tendríamos que considerar la existencia de un clima emocional que permitiera la interacción funcional entre padre e hijos para poder alcanzar dichos objetivos. Para esto, los estilos y las prácticas parentales se han convertido en un vehículo para crear dicho clima emocional donde se manifiestan las conductas de los padres, se facilita la expresión de las emociones y permite el establecimiento de las reglas y normas. Lo anterior es de suma importancia para el desarrollo emocional, psicológico y social del niño ya que permea su desarrollo hasta su adolescencia.

Desde hace una década, Steinberg y Darling (1992) sugirieron establecer una clara distinción entre los estilos de crianza y las prácticas parentales utilizadas por los padres para la socialización de sus hijos. Los estilos de crianza pueden ser entendidos como una constelación de actitudes que los padres realizan para conseguir ciertos objetivos con respecto a la educación de los hijos y que, en su conjunto, crean un clima emocional en el que se ponen de manifiesto los comportamientos de los padres. La investigación sobre este componente se ha centrado en tres unidades: la relación emocional entre los padres e hijos, las prácticas y comportamientos de los padres y, por último, su sistema de creencias (Darling \& Steinberg, 1993).

En cambio, las prácticas parentales incluyen tanto las conductas, a través de las cuales los padres desarrollan sus propios deberes de paternidad, como cualquier otro tipo de comportamiento, es decir, gestos, cambios en el tono de voz, expresiones espontáneas de afecto, entre otros elementos. Dichos comportamientos parentales individuales son parte de un contexto de muchos otros comportamientos y, por lo tanto, la influencia de una conducta individual no puede ser analizada fácilmente de manera independiente, ni tampoco es factible determinar su influencia en el desarrollo del niño, pues se perdería entre la complejidad de otros atributos parentales; de ahí la importancia de estudiar las prácticas en su conjunto, de manera bidireccional, dada la modelación que sufren al incidir en el desarrollo del niño. Tales circunstancias han llevado a que el estilo parental sea usado como un constructo que aglutina y resume los resultados de muchos análisis realizados sobre prácticas parentales específicas, más que para referirse a él como una entidad en sí misma (Rayas-Trena, 2008).

Por su parte, Baumrind (1991) desarrolló un modelo teórico que incorporaba los procesos emocionales y conductuales en función de la dimensión del control. Este concepto de control parental tenía una definición que contrastaba con lo que hasta el momento se había utilizado: castigo físico, consistencia de castigo y rectitud. El autor lo utilizó para referirse a los intentos de los padres para integrar al niño dentro de la familia y la sociedad además de cumplir con los 
comportamientos socialmente aceptados. Con base en este concepto diferenció, en principio, tres tipos de control parental cualitativamente diferentes: autoritarios, permisivos y autoritativos. Estas dimensiones englobaban desde el uso de las técnicas de disciplina hasta las ideologías que las justificaban, de manera que la influencia de algún aspecto del estilo parental es dependiente de la combinación de todos los otros aspectos.

La tabla 1 ilustra las características de los padres de acuerdo al estilo parental en el que se integran. De esta manera, los padres autoritarios son padres con altas demandas y escasa

\section{Tabla 1.}

Características de los padres según su estilo educativo

\begin{tabular}{|c|c|}
\hline \multicolumn{2}{|r|}{ Características de los padres según su estilo educativo } \\
\hline 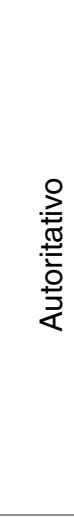 & $\begin{array}{l}\text { - } \quad \text { Prestan atención a las demandas y preguntas de sus hijos y muestran interés. } \\
\text { - } \quad \text { Fanifiestan una combinación de afecto y apoyo con ciertas dosis de control y democracia. } \\
\text { - } \quad \text { Son controladores y exigentes en sus demandas, pero al mismo tiempo se muestran cariñosos, } \\
\text { - } \quad \text { Estabonables y comunicativos. } \\
\text { - } \quad \text { Sus prácticas disciplinarias se orientan más hacia la inducción que hacia el castigo. } \\
\text { - } \quad \text { El castigo es razonado y verbal, pero no físico. } \\
\text { - } \quad \text { Ea comunicación es efectiva y bidireccional, sin órdenes ni gritos. } \\
\text { - } \quad \text { Muestran pocas conductas problemáticas (adicciones, violencia...), bajos niveles de estrés y } \\
\text { un clima familiar estable. }\end{array}$ \\
\hline $\begin{array}{l}\frac{0}{\frac{0}{2}} \\
\frac{\pi}{2} \\
\frac{0}{3} \\
\frac{1}{2}\end{array}$ & $\begin{array}{l}\text { - } \quad \text { Combinan altos niveles de exigencia y control con escasa sensibilidad o responsividad. } \\
\text { - } \quad \text { No consideran las peticiones de sus hijos ni responden a sus demandas. } \\
\text { - } \quad \text { Proporcionan ambiente ordenado, con reglas claras dictadas por los padres. } \\
\text { - } \quad \text { Son más restrictivos, convencionales y prestan escaso apoyo emocional al hijo. } \\
\text { - } \quad \text { Presentan más problemas de conducta e insatisfacción en la pareja. }\end{array}$ \\
\hline 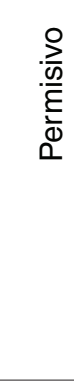 & $\begin{array}{l}\text { - Combinan baja dosis de control y exigencia con relativa sensibilidad hacia las necesidades } \\
\text { - } \quad \text { Sel niño. } \\
\text { - No muestran autoridad frente a sus hijos. } \\
\text { - No demandan conductas maduras de sus hijos y evitan el enfrentamiento con éstos. } \\
\text { - La comunicación es poco efectiva y unidireccional. } \\
\text { - Mantienen gran flexibilidad en el seguimiento de reglas, dificultando la asunción de } \\
\text { obligaciones por parte del niño. } \\
\text { - No existen reglas claras y el ambiente familiar es desorganizado. }\end{array}$ \\
\hline 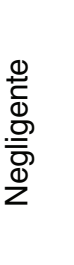 & $\begin{array}{l}\text { - } \quad \text { Ausencia de demandas y de responsividad hacia la conducta de los hijos. } \\
\text { - } \quad \text { Falta de estructuración, control y apoyo de las conductas del niño. } \\
\text { - } \quad \text { Presentan problemas de conducta. } \\
\text { - } \quad \text { Proporcionan un ambiente familiar desorganizado. } \\
\text { - } \quad \text { Son altamente vulnerables a la ruptura familiar. }\end{array}$ \\
\hline
\end{tabular}

Fuente: Baumrid (1971, 1991). 
responsividad, valoran la obediencia y creen en la restricción de la autonomía de los hijos, los permisivos son padres poco demandantes y poco responsivos; los padres autoritativos son muy demandantes y muy responsivos, intentan dirigir las actividades del hijo de modo racional. Más adelante se incorporó la dimensión de padres negligentes, quienes tienen escasa responsividad y son poco demandantes. De acuerdo con esto, los padres autoritativos agrupan un conjunto de características que abarcan el apoyo emocional, la posibilidad de autonomía y la comunicación bidireccional, lo que permite a los hijos desarrollar competencia instrumental, caracterizada por cooperación con adultos y compañeros, independencia responsable, madurez psicosocial y éxito académico.

El término de responsividad fue propuesto en primera instancia por Maccoby y Martin (1983), al fusionar dos dimensiones a las que llamaron afecto/comunicación en contraste con la dimensión de control/exigencia, mismas que fueron integradas al modelo propuesto por Baumrind. Estos estilos quedan esquematizados en la siguiente tabla.

Maccoby y Martín comprobaron que lo que ayuda al desarrollo del niño y al sentido de inde- pendencia y autonomía no es el control sino la comunicación bidireccional y un alto componente de afecto en las interacciones padres-hijos. En este sentido, el afecto es operacionalizado como la capacidad de los padres para mostrar su sensibilidad y adaptarse a las señales del niño, sus estados y necesidades. Por otro lado, la exigencia se refiere a la voluntad de los padres para actuar como un agente socializador, haciendo énfasis en el cumplimiento de normas disciplinarias.

A partir de los dos modelos clásicos expuestos sobre los estilos y prácticas parentales se han derivado investigaciones que dan muestra de su influencia en el desarrollo de los niños y de los adolescentes. Por ejemplo, se sabe que el conjunto de actitudes y conductas de los padres autoritativos incluye el razonamiento inductivo como técnica de disciplina. El uso de estos criterios y razonamientos inductivos en la práctica educativa se relaciona con el comportamiento pro social y, en concreto, con la internalización moral (Hoffman, 1982, 1990), que actuaría como un factor de protección de problemas de comportamiento y depresión durante la adolescencia.

Lo anterior se demuestra en el estudio de Mester, Tur, Samper y Cortés (2007), quienes

Tabla 2.

Tipología de estilos educativos de Maccoby y Martín

\begin{tabular}{cc}
\hline \multicolumn{1}{c}{ Alto } & \multicolumn{1}{c}{ Afecto y comunicación } \\
\hline & Afecto y apoyo explícito, Afecto controlado y no \\
aceptación e interés explícito, distanciamiento, \\
por las cosas del hijo frialdad en las relaciones, \\
y sensible ante sus hostilidad o rechazo \\
necesidades
\end{tabular}

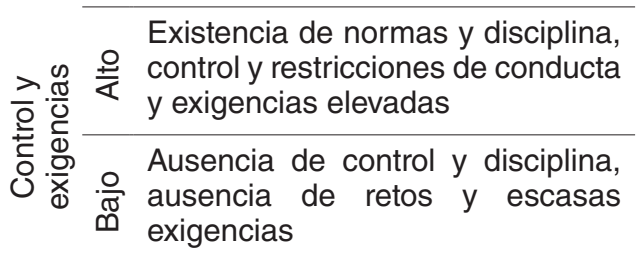

Fuente: Raya Trenas (2008).
Autoritativo

Autoritario

Permisivo

Negligente 
encontraron en población adolescente, que la capacidad de mantener comportamientos empáticos de parte de los padres, como mostrarse sensibles hacia las necesidades de sus hijos y atribuirles intenciones benévolas a sus comportamientos fueron los principales predictores del comportamiento pro social. En estudios realizados por Rodrigo, Máiquez, García, Mendoza et al. (2004), se confirmó que la convivencia familiar, percibida por el adolescente y caracterizada por el afecto y apoyo emocional, especialmente por parte de su madre, junto con la estimulación de la autonomía del hijo, alcanza un poder predictor del comportamiento pro social.

Los resultados de estas investigaciones indican que el uso del estilo parental, en este caso el autoritativo, comprueba que muchas veces la clara expresión de los deseos y conductas, la manifestación de afecto y una comunicación bidireccional influyen en la apertura de los hijos hacia los intentos de socialización de los padres, y aumentan, a su vez, la efectividad de la paternidad y la habilidad de los padres para actuar como agentes de socialización.

Asimismo, una crianza plena de calidez y apoyo emocional se ha asociado con un buen ajuste emocional de los hijos. Desde un punto de vista cognitivo, los estilos de crianza consisten en una colección de conductas que llevan información a los niños, quienes la internalizan para formar su auto concepto (Frías, Fraijo \& Tapia, 2011), de tal forma que éstos aprenden a construir su realidad a través de experiencias tempranas con el ambiente. Si la información que aprecian de parte de sus padres es empática y relacionada con la aceptación se propiciaría una percepción de competencia personal o control sobre su ambiente, asociada a una autovaloración positiva y de capacidad para afrontar situaciones estresantes (Arroyo, 2009).

Por el contrario, muchos de los problemas propios de la adolescencia están relacionados con la falta de afecto y apoyo, además, en bastantes casos son una reacción a una serie de normas o límites establecidos de forma unilateral o autoritaria por unos padres poco dialogantes (Oliva, 2006). Incluso, estudios de estilo de crianza con adolescentes que consumen drogas (Sánchez, Zapata, León \& Fabián, 2008) han demostrado que el consumo de drogas en adolescentes es elevado con el estilo de crianza negligente y permisible entre consumidores.

Por otra parte, se conoce desde hace tiempo que los desenlaces negativos como la ansiedad y la depresión de los hijos se asocian con las transacciones defectuosas entre padres e hijos. Hay padres que no sólo no fomentan el manejo adecuado de las emociones ante sucesos estresantes, sino que ellos mismos son los que los provocan. Las transacciones defectuosas son propias del rechazo, el castigo corporal y de la ausencia de sus contrapartes positivas (Aracena, Castillo, Haz et al., 2000). Los estudios demuestran que los niños rechazados por sus padres muestran más problemas psicológicos y sociales que los niños que sí son aceptados (Lila \& Gracia, 2005). A mayor rechazo percibido en la niñez y adolescencia resulta peor el autoconcepto en la adultez temprana, lo cual provoca mayor vulnerabilidad al uso de sustancias adictivas como el alcohol (Mathews \& PilIon, 2004).

Hay que destacar que los estilos de crianza varían de acuerdo con la cultura e incluso entre los diversos contextos de una misma cultura, ya que ésta genera creencias sobre la efectividad de una serie de estrategias educativas, esto influirá en los estilos de crianza que los padres adopten. Resulta indispensable saber que los estudios transculturales acerca de la familia y su papel socializador muestran que México es una cultura colectivista (Díaz-Guerrero, 2001), donde el poder está asociado a diversos conceptos, entre ellos el de autoridad y mando con carácter impositivo, caracterizado por una modalidad dominio-sumisión. Se espera que los hijos no cuestionen la autoridad de los padres sino que les deban obediencia. Esto concuerda con los resultados encontrados por Flores, 
Cortes, Campos y García (2011), según los cuales las prácticas educativas que predominaron en una población adolescente de los estados de Yucatán e Hidalgo, fueron los regaños y los castigos ejercidos tanto por los padres como por las madres. Caso contrario fueron los estudios realizados en el medio rural del estado de México, Saltillo y Monterrey, donde las prácticas educativas se caracterizaban más por una actitud responsiva, con privilegio de la dimensión afectiva sobre el componente de control (Oudhof, González \& González, 2011; González, Garza \& Hernández, 2011 y Moral, 2011). Lo anterior nos induce a pensar que la familia está siendo impactada por los avances en la tecnología, educación y servicios, circunstancia que repercute a su vez en sus creencias, valores y dinámica familiar, además, de la evolución en los roles y estereotipos de género.

Incluso se puede pensar que las prácticas parentales en el contexto mexicano no corresponden a la clasificación que propuso en su momento Baumrind, tal como lo demuestra el estudio de Gaxiola, Frías, Cuamba, Franco y Olivas (2006), ya que al momento de validar un cuestionario de prácticas parentales construidos de acuerdo con la clasificación del autor, se encontró que el instrumento evaluado presentó dos factores en los sujetos estudiados, los estilos autoritarios y autoritativos, en lugar de los tres factores propuestos por su autor. La falta de supervisión y el ignorar el mal comportamiento, originalmente incluidos en el factor permisivo, se integraron al factor autoritario, y la falta de confianza correspondió al factor autoritativo en la muestra estudiada, es decir, las madres confundieron la autoridad democrática con la permisividad; también hay que destacar en este estudio que el diseño utilizado para medir las variables fue cuantitativo.

Tal preferencia deja una línea de investigación cualitativa muy importante sobre el fenómeno de las prácticas parentales en la cultura mexicana si tomamos en cuenta la necesidad de dar mejores explicaciones a estos cambios que, aparentemente, pueden ser contradictorios a partir del análisis e identificación de diferencias según el género de pertenencia de los adolescentes y la diferenciación entre padre y madre, a través de la búsqueda del significado e intención otorgada al comportamiento parental. Cuestión que a través del análisis cualitativo puede desembocar en resultados relevantes.

\section{Método}

\section{Participantes}

El procedimiento de selección de los participantes se llevó a cabo gracias al consentimiento de las autoridades educativas de una zona escolar del sector oriente de la ciudad de Saltillo, Coahuila. Fueron 133 alumnos de nivel secundaria, de este grupo de participantes $55.6 \%$ son mujeres y el $44.4 \%$ son hombres. No hubo diferencias significativas en la proporción de hombres y mujeres participantes, $\chi 2=1.939$, $\mathrm{gl}=1, \mathrm{p}=.164$. Las edades de los participantes van de los 12 a los 15 años; la media de edad fue de 14 años con una desviación estándar de 1. El $81.2 \%$ vive con ambos padres, $15.8 \%$ vive sólo con su mamá y el $3 \%$ reportó vivir con otra persona como tíos y abuelos.

\section{Procedimiento}

Se aplicó un cuestionario de 10 preguntas abiertas, desarrollado particularmente para esta investigación (Lozano Razo et. al., 2011; González, Garza \& Hernández, 2011; Frías Armenta, Fraijo Sing \& Tapia Fonllen, 2011; Moral de la Rubia, 2011; Oudhof, González \& González-Arratia, 2011; Flores Galaz et. al, 2011), se tomaron como base para el análisis las dos preguntas acerca de las prácticas educativas de los padres y las madres, con el objetivo de establecer las discrepancias entre hombres y mujeres sobre las prácticas de sus padres y, asimismo, diferenciar entre padre y madre el comportamiento ante los hijos. 


\section{Análisis de datos}

El análisis del contenido de las respuestas se hizo mediante el programa Atlas Ti versión 6.1. El procedimiento de análisis del contenido de las respuestas fue realizado según el género de los respondientes. El modelo teórico utilizado, inicialmente, fue el propuesto por Andrade y Betancourt (2011) en su Escala de Prácticas Parentales en Adolescentes, la cual considera para las mamás cinco factores: comunicación, control psicológico, imposición, control conductual y autonomía. Para los padres son cuatro factores: comunicación/control conductual, control psicológico, autonomía e imposición; sin embargo, se hace la aclaración de que a partir de la información que emergió del análisis de las respuestas de los participantes sólo resultaron pertinentes dos factores, tanto para padres como madres. El análisis cualitativo a partir de las categorías establecidas en un análisis cuantitativo se hizo para que los factores propuestos por Andrade y Betancourt fungieran como una categoría central que permitiera dar un mejor sentido a los datos obtenidos (Rodríguez, Gil \& García, 1996). El análisis de los datos se realizó por sexo de los participantes y según la figura parental, se decidió abordarlo desde esta perspectiva, debido a que diferentes estudios (Leaper \& Friedman, 2007; Calvete, Gámez-Guadix \& Orue, 2010) han demostrado diferencias por sexo en cuanto a los estilos de socialización parental con niños y adolescentes; además de que estas diferencias han sido analizadas desde la perspectiva cuantitativa sin entrar en un análisis de contenido que permita identificar el significado e intención en los procesos de socialización y procesos educativos en la familia.

A partir de estos factores fueron clasificadas las respuestas a dos preguntas del instrumento sobre las cuales se hizo énfasis en el estudio: ¿qué hace tu mamá para educarte? y ¿qué hace tu papá para educarte? El análisis de las respuestas a estas preguntas se realizó por género. El análisis de los resultados se llevó a cabo a partir de generar dos familias para hombres y dos para mujeres.

\section{Resultados}

Las familias construidas para las respuestas de las mujeres fueron control psicológico y control conductual, éstas coinciden con las propuestas por Andrade y Betancourt (2008). El control psicológico está relacionado con las actitudes de devaluación y chantaje que realizan los padres hacia los hijos (Segura-Celis, Vallejo, Osorno, Rojas \& Reyes-García, 2011). El control psicológico en las mujeres adolescentes se expresó mediante prácticas como el regaño verbal, el cual forma parte de las estrategias de supervisión de los padres a los hijos, al preguntar sobre tareas y cuestiones relacionadas con los estudios y la labor de corregir errores del hijo. La labor de supervisión forma parte, también, del seguimiento que los padres dan a las actividades educativas de los hijos. La información sobre dichas ocupaciones de los hijos se obtuvo mediante la comunicación a través de pláticas y charlas con fines de supervisión; estas prácticas, desde la perspectiva de las mujeres, son expresiones de los valores que los padres tratan de inculcar.

El control conductual es la segunda familia identificada en las respuestas de las mujeres. Esta variable implica la supervisión y conocimiento de las actividades de los hijos, cuyos efectos son benéficos para ellos (Barber \& Harmon, 2002; Steinberg \& Silk, 2002 citados por Segura-Celis et. al., 2011). Las respuestas de las mujeres agrupadas en esta familia correspondieron a cinco códigos (categorías): protección y cuidado, plática, valores, supervisión y educación. La protección y el cuidado se evidenció como práctica parental a través de la supervisión que tiene el padre mediante las pláticas con su hijo; el contenido de las mismas va dirigido a temas escolares, apoyo en decisiones que tiene que tomar el adolescente 


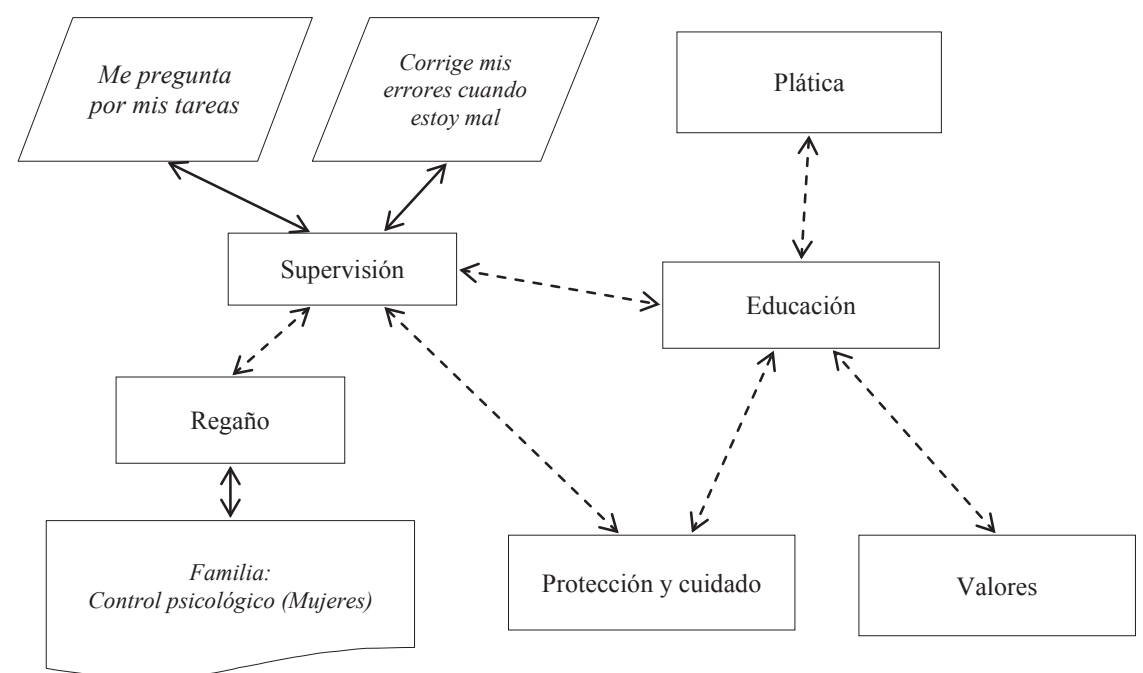

Figura 1. Red de trabajo para la Familia Control Psicológico construida con las repuestas de las mujeres adolescentes. La línea punteada significa asociación entre los códigos o categorías, la línea completa implica propiedad.

Fuente: elaboración propia.

y brindar consejo. Otro aspecto que conforma el control conductual en las respuestas fue el referente a las prácticas de reproducción de valores, en donde las mujeres respondieron que sus padres las educan enseñándoles sus valores o reproduciendo los valores que a ellos les inculcaron, además de la dimensión moral y ética expresada con estrategias como enseñar lo que es bueno y lo que es malo y lo que está bien y lo que está mal. Asimismo, la supervisión como parte del control conductual incluyó las prácticas parentales como prestar atención al hijo, corregirlo, establecimiento de límites y reglas, por ejemplo, establecer claramente sus obligaciones y derechos y la percepción de cuidado hacia el hijo. El quinto código asignado a esta familia correspondió a las prácticas orientadas a la educación, en donde el padre y madre de familia manifiestan su apoyo en todo lo que tenga que ver con el estudio, desde pagos, manutención, traslado y ayuda con tareas.

Del análisis de las prácticas educativas de los hombres resultaron dos familias: control psicológico y control conductual; el resultado coin- cidió aparentemente, con el de las mujeres. Sin embargo, al revisar las prácticas en particular y los códigos que componen las mencionadas familias, se encontró que hay tres códigos: escuela, supervisión y apoyo, además de consejo. El código de escuela es similar en contenido al de las mujeres, refleja prácticas como apoyo en tareas, ayuda con materiales, pago de colegiaturas y traslado hacia la escuela. La supervisión se evidenció mediante prácticas como un cuestionamiento constante sobre las obligaciones del alumno. El código supervisión está relacionado con el cogido valores que incluye prácticas como ayuda con tareas y enseñanza de valores además de mostrar lo correcto e incorrecto en las acciones.

La segunda familia del análisis de las respuestas de los hombres también fue el control psicológico el cual se compone de cuatro códigos: estricto, corrige, regaño, castigo y castigo físico, estos dos últimos se encuentran asociados; el castigo está relacionado con una actitud estricta del padre hacia el hijo. Lo anterior lleva al padre y madre de familia a asumir prácticas 
educativas orientadas a corregir comportamientos no deseados del adolescente tanto a través de amonestaciones verbales como son los regaños y llamados de atención. Asimismo, estas conductas tratan de eliminarse mediante el sometimiento con castigos y sanciones, por ejemplo, suspender el uso de aparatos electrónicos, el derecho a realizar actividades de ocio y la aplicación de castigos físicos como golpes.

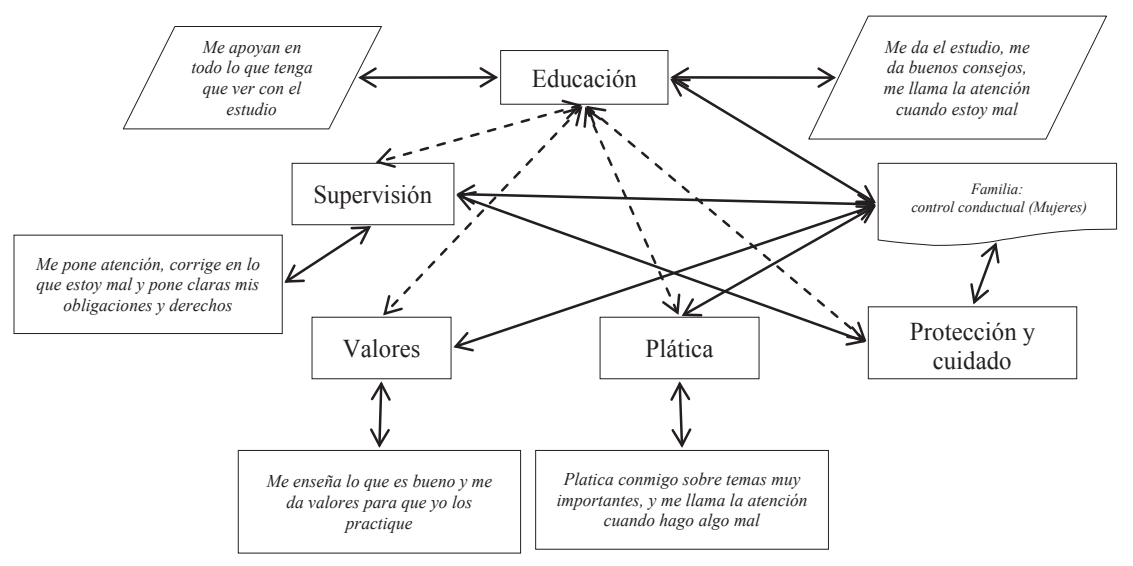

Figura 2. Red de trabajo para la Familia Control Conductual construida con las repuestas de las mujeres adolescentes. La línea punteada significa asociación entre los códigos o categorías, la línea completa implica propiedad.

Fuente: elaboración propia.

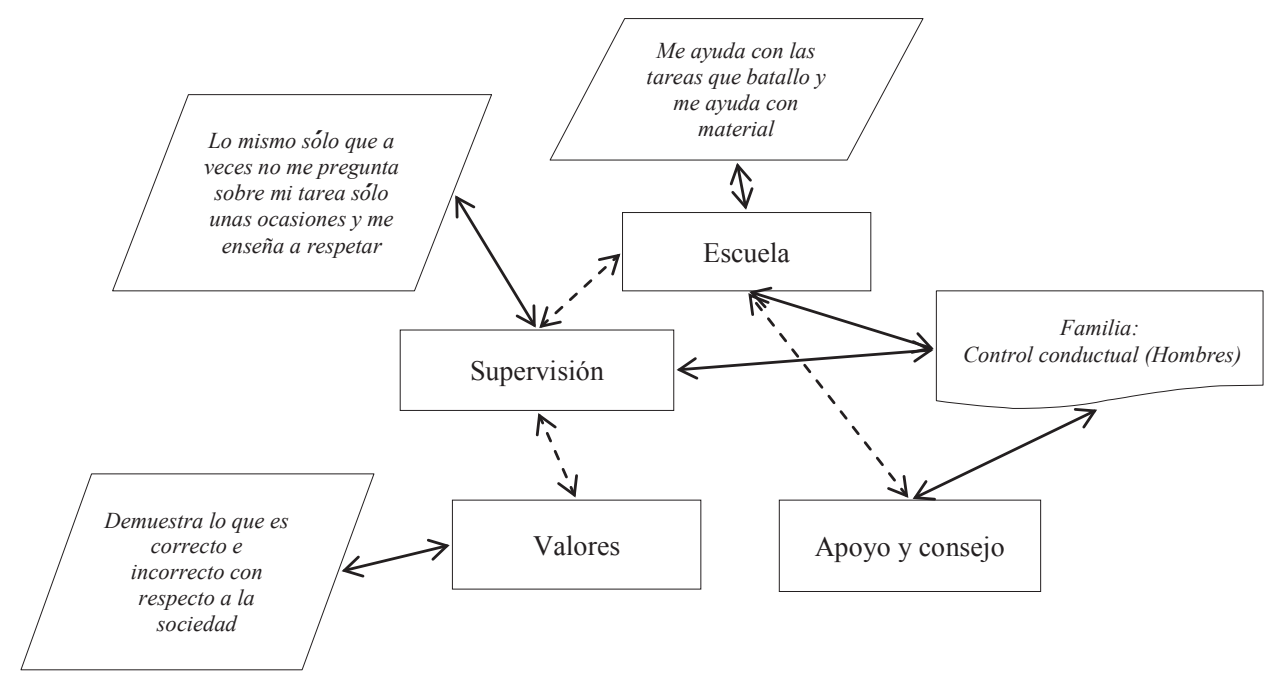

Figura 3. Red de trabajo para la Familia Control Conductual construida con las repuestas de los hombres adolescentes. La línea punteada significa asociación entre los códigos o categorías, la línea completa implica propiedad.

Fuente: elaboración propia. 


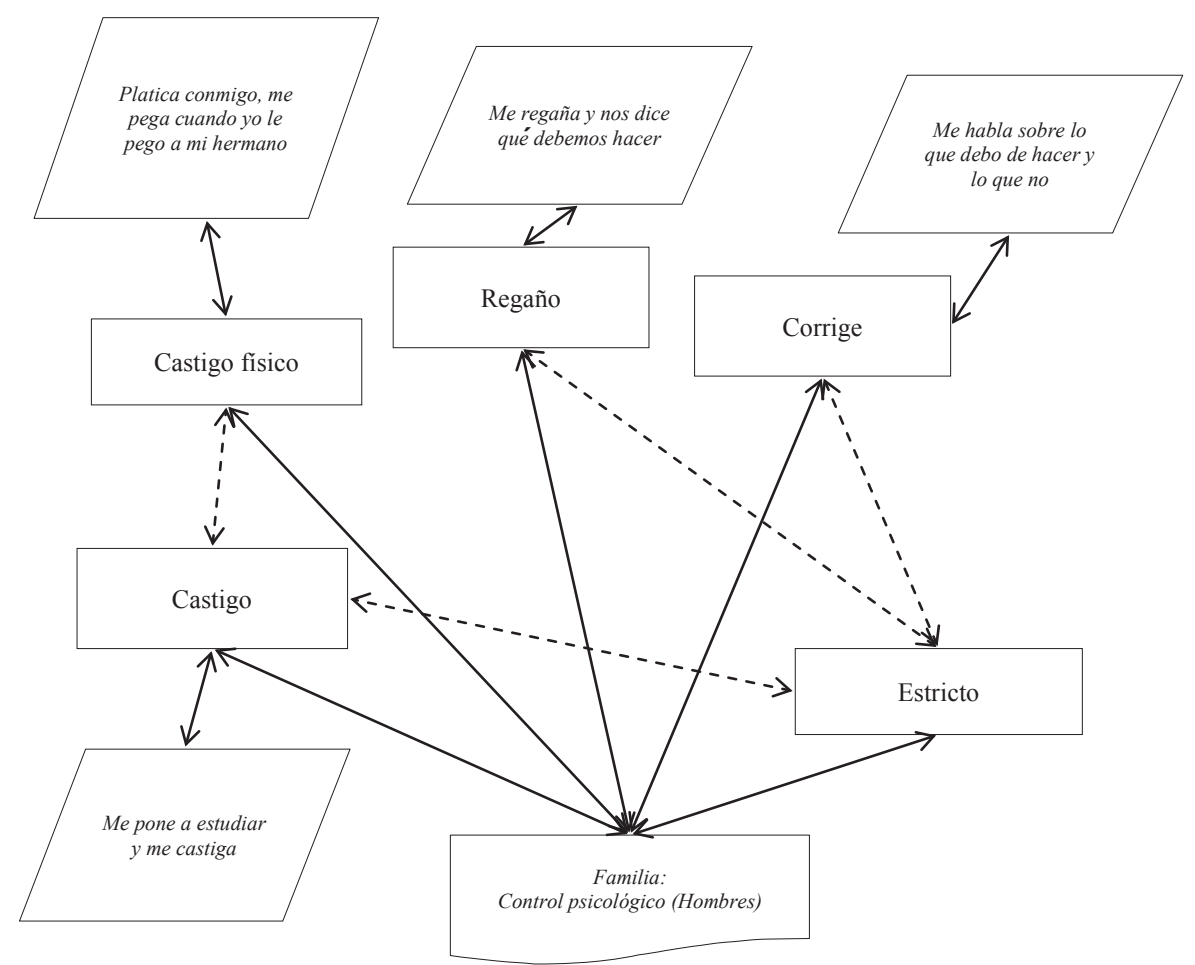

Figura 4. Red de trabajo para la Familia Control Psicológico construida con las repuestas de los hombres adolescentes. La línea punteada significa asociación entre los códigos o categorías, la línea completa implica propiedad.

Fuente: elaboración propia.

\section{Discusión}

El propósito de este artículo fue identificar las diferencias cualitativas de las prácticas educativas en la familia a la luz del modelo factorial presentado por Andrade y Betancourt (2008), validado en adolescentes mexicanos por Segura-Celis et al., (2011). A partir de las respuestas de carácter cualitativo dadas a las dos preguntas sobre lo que hace el padre y madre para educar a su hijo desde la perspectiva del adolescente se encontraron puntos de convergencia y divergencia. En primer término, las prácticas parentales orientadas al control psicológico y conductual en el adolescente están presentes tanto en hombres como en mujeres.
Se encontraron diferencias cualitativas en las prácticas específicas y los códigos en donde se agruparon. En las mujeres, el control psicológico sólo se expresó mediante los llamados de atención de forma verbal, aun y cuando éste tuvo relación con las prácticas de supervisión, educación, valores y protección y cuidado. En el caso de los hombres, el control psicológico presentó una estructura más compleja, en donde aparece el castigo físico, verbal, la corrección y la disciplina como prácticas parentales. Por otro lado, el control conductual en las mujeres resultó más complejo que en los hombres, la diferencia más marcada correspondió a prácticas de comunicación y diálogo con los hijos, las cuales están presentes en las mujeres y no en los hombres, además de la percepción de protección y cuidado. 
En relación con el modelo de los estilos educativos de Maccoby y Martin (1983), la estructura de las prácticas educativas corresponde a la dimensión de control y exigencias a un nivel alto, ya que existen las normas en las familias, el control se ejerce mediante la dimensión moral y ética, además de las restricciones de conductas inadecuadas o equivocadas, en donde el papel del padre y de la madre de familia es el de corregir.

En los aspectos de afecto y comunicación, hubo expresiones de apoyo explícito, sobre todo en la percepción de las mujeres; esto se demostró con las prácticas educativas de la categoría de apoyo y protección y cuidado. De igual modo, la existencia de una comunicación bidireccional es un elemento que añade consistencia a la clasificación de éstas prácticas parentales, las ubica en un estilo de crianza autoritativo para el caso de las respuestas de las mujeres. Dichas formas de relación han demostrado un impacto positivo en la construcción de un autoconcepto sólido, que permite a los adolescentes afrontar situaciones estresantes (Arroyo, 2009), así como la generación de conductas pro sociales que actúan como un factor de protección de problemas de comportamiento y depresión (Mester et al., 2007); relaciones que se han encontrado en diferentes muestras de países occidentales, incluido México (Oudhof, González \& González, 2011; González, Garza \& Hernández, 2011; Moral, 2011).

En los hombres se presentó un elemento diferenciador en las prácticas educativas enunciadas referente al castigo físico, el cual fue un elemento aún presente en la dinámica padrehijo en la etapa adolescente. Las mujeres no reportaron más que la amonestación verbal como método de corrección. Los hombres reportaron tanto el castigo físico como el verbal en sus respuestas, aunque no se precisó el nivel de severidad del mismo ni las características particulares de su aplicación. Este rasgo acerca las prácticas parentales en hombres a un estilo de crianza similar al autoritario. Estas formas de relación han demostrado tener un efecto negativo en el bienestar psicológico de los hijos, potencializando la exteriorización de reacciones emocionales exageradas, acompañadas de una falta de control, la cual se puede traducir en el consumo temprano de drogas y alcohol (Mathews \& Pillon, 2004), además de la presencia de problemas psicológicos como la depresión y la ansiedad (Lila \& Gracia, 2005).

Las diferencias cualitativas encontradas por género en las prácticas parentales percibidas por los hombres y por las mujeres que participaron en el estudio sirven como línea de investigación para el análisis a profundidad de las prácticas parentales, de los rasgos y el significado del proceso de socialización en la familia; sobre todo partiendo de la extensa revisión cuantitativa del fenómeno de la crianza (Leaper \& Friedman, 2007; Calvete, Gámez-Guadix \& Orue, 2010; Musitu \& García, 2004; Rodrigo et al., 2004).

Como apunte final se puede destacar el contraste de los resultados de este estudio con el antecedente en tres municipios del estado de México, Coahuila y Nuevo León, donde la dimensión afectiva fue un elemento importante; sin embargo, en análisis por género de estas prácticas parentales resaltó la dimensión de control, sobre todo en los hombres. Esto nos lleva a pensar en el papel de los estereotipos en la cultura mexicana y su influencia en las prácticas de crianza, donde se justifica el trato más enérgico y poca comunicación hacia los varones, y un trato de mayor protección y cuidado, así como una comunicación más abierta que permita la expresión de las emociones en las mujeres.

Posterior a este análisis y su posible aplicación, hay que tomar en cuenta las limitaciones del estudio y del diseño cualitativo en cuanto a la representatividad estadística, por un lado y, por el otro, la dificultad para identificar variables que discriminen o hagan diferencia en los resultados, por ejemplo, variables sociodemográficas. 
Andrade, P., \& Betancourt, D. (2008). Prácticas parentales: Una medición integral. En S. Rivera Aragón, R. Díaz-Loving, R. Sánchez-Aragón \& I. Reyes-Lagunes (Eds.). La Psicología Social en México (561-565). México: AMEPSO.

Aracena, M., Castillo, R., Haz, A., Cumsille, F., Muñoz, S., Bustos, L. \& Román, F. (2000). Resiliencia al maltrato infantil: Variables que diferencian a los sujetos que maltratan y no maltratan físicamente a sus hijos. Revista de Psicología de la Universidad de Chile, 9(1), $1-22$.

Arroyo, M. (2009). El autoconcepto durante los años preescolares y escolares. Revista Digital Transversalidad Educativa, 23(6), 4-10.

Baumrind, D. (1971). Current patterns of parental authority. Developmental Psychology Monograph, 4(1), 1-103.

Baumrind, D. (1991). Parenting styles and adolescent development. En J. Brooks-Gunn, R. Lerner \& A.C. Petersen (Eds.), The Encyclopedia of Adolescent. (746-758). New York: Garland.

Calvete, E., Gámez-Guadix, M. \& Orue, I. (2010). El Inventario de Dimensiones de Disciplina (DDI), versión niños y adolescentes: Estudio de prácticas de disciplina parental desde una perspectiva de género. Anales de Psicología, 26(2), 410-418.

Darling, N. \& Steinberg, L. (1993). Parenting style as context: An integrative model. Psychological Bulletin, 113(3), 487-496.

Díaz Guerrero, R. (2001). Psicología del mexicano, descubrimiento de la etnopsicología. México: Trillas.

Flores-Galaz, M.M., Cortés-Ayala, M.L., Campos-Mota, M.J. \& GarcíaMerz, M. (octubre, 2011). Crianza y obediencia en adolescentes de Yucatán e Hidalgo. En M. Flores Galaz (Coord.), La crianza paterna: la visión de los adolescentes y padres de diferentes ecosistemas. Simposio llevado a cabo en el Congreso Mexicano de Psicología, Cancún, Quintana Roo.

Frías-Armenta, M., Fraijo-Sing, B. \& Tapia-Fonllem, C.C. (octubre, 2011). Percepción de las prácticas de crianza de los niños. En M. Flores Galaz (Coord.), La crianza paterna: la visión de los adolescentes y padres de diferentes ecosistemas. Simposio llevado a cabo en el Congreso Mexicano de Psicología, Cancún, Quintana Roo.

Gaxiola, J., Frías, M., Cuamba, N., Franco, J. \& Olivas, L. (2006). Validación del cuestionario de prácticas parentales en una población mexicana. Enseñanza e Investigación en Psicología, 11(1), 115-128.

González-Tovar, J., Garza-Sanchez, I. \& Hernández-Montaño, A. (octubre, 2011). Análisis padre-hijo de las prácticas parentales en adolescentes. En M. Flores Galaz (Coord.), La crianza paterna: la visión de los adolescentes y padres de diferentes ecosistemas. Simposio llevado a cabo en el Congreso Mexicano de Psicología, Cancún, Quintana Roo.

Leaper, C. \& Friedman, C.K. (2007). The socialization of gender. En J.E. Grusec, \& P.D. Hastings (Eds.), Handbook of socialization: Theory and research (561-587). New York: Guilford Press.

Lozano-Razo, G., García-Sánchez, M.D., Jiménez-López, M.D., LuisDelgado, O.E., Miramontes-Zapata, S., Pacheco-Medina, F. J., (Octubre, 2011). Afecto y crianza: Experiencia de hombres y mujeres adolescentes Zacatecanos respecto a la demostración de afecto materno y paterno. En M. Flores Galaz (Coord.), La crianza paterna: la visión de los adolescentes y padres de diferentes ecosistemas. Simposio llevado a cabo en el Congreso Mexicano de Psicología, Cancún, Quintana Roo.

Lila, M. \& García, E. (2005). Determinantes de la aceptación-rechazo parental. Psicothema, 17(5), 107-111.

Maccoby, E. \& Martin, J. A. (1983). Socialization in the context of the family: Parent-child interaction. En P. H. Mussen \& E. M. Hetherington (Eds.), Handbook of child psychology: Vol. 4. Socialization, personality and social development (1-101). New York: Wiley.

Mathews, I. \& Pillon, S. (2004). Factores protectores y de riesgo asociados al uso de alcohol en adolescentes hijos de padres alcohólicos, en el Perú. Revista Latino-Americana de Enfermagem, 12(spe), 359-368.

Mester, M., Tur, A., Samper, M. \& Cortés, M. (2007). Estilos de crianza en la adolescencia y su relación con el comportamiento pro social. Revista Latinoamericana de Psicología, 39(2) ,123-131.
Moral de la Rubia, J. (octubre, 2011). Prácticas de crianza y percepción de su rol en padres de adolescentes. En M. Flores Galaz (Coord.), La crianza paterna: la visión de los adolescentes y padres de diferentes ecosistemas. Simposio llevado a cabo en el Congreso Mexicano de Psicología, Cancún, Quintana Roo.

Musitu, G. \& García, J.F. (2004). Consecuencias de la socialización familiar en la cultura española. Psicothema, 16(2), 288-293.

Oliva, A. (2006). Relaciones familiares y desarrollo adolescente. Anuario de Psicología, 37 (3), 209-223.

Oudhof van Barneveld, H., González, S., González-Arratia, N.I. (Octubre, 2011). Prácticas de crianza en padres y madres del medio rural del Estado de México. En M. Flores Galaz (Coord.), La crianza paterna: la visión de los adolescentes y padres de diferentes ecosistemas. Simposio llevado a cabo en el Congreso Mexicano de Psicología, Cancún, Quintana Roo.

Papalia, D \& Wendwoks, O. (2009). Psicología del desarrollo de la infancia a la adolescencia (11를. ed.). México: McGraw-Hill.

Parra, A. \& Oliva, A. (2002). Comunicación y conflicto familiar durante la adolescencia. Anales de Psicología, 18(2) 215-231.

Raya-Trenas, A. (2009) Estudio sobre los estilos educativos parentales y su relación con los trastornos de conducta de la infancia (Tesis de doctorado inédita). Universidad de Córdova. Córdova, España. Recuperada de: helvia.uco.es/xmlui/handle/10396/2351.

Rodríguez-Gómez, G., Gil-Flores, J. \& García-Jiménez, E. (1996). Metodología de la investigación cualitativa. Madrid: Aljibe.

Rodrigo, M., Máiquez, L., García, M., Mendoza, R., Rubio, A., Martínez, A. \& Matín, J. (2004). Relaciones padres-hijos y estilos de vida en la adolescencia. Psicothema, 16(2) 203-210.

Sánchez, E., Zapata, K., León, M., \& Fabián, E. (2008). Crianza y consumo de drogas en población de adolescentes de un suburbio de Lima Ciudad. Revista Enfermería Herediana, 1(1), 57-61.

Segura-Celis, H.B., Vallejo-Casarín, A.G., Osorno-Munguía, J.R., RojasRivera, R.M., \& Reyes-García, S.I. (2011). La escala de prácticas parentales de Andrade y Betancourt en adolescentes veracruzanos. Revista Educación y Desarrollo, (18), 67-73.

Steinberg, L., \& Darling, N. (1992). Impact of parenting practices on adolescent achievement: Authoritative parenting, school involvement, and encouragement to succeed. Child Development, 63(5), 1266-1281. 